Published in final edited form as:

Exp Eye Res. 2011 October ; 93(4): 329-330. doi:10.1016/j.exer.2010.06.008.

\title{
Focus on Molecules: Syndecan-1
}

\author{
Yinghui Zhang ${ }^{\mathrm{a}}$, Robert L. McKown ${ }^{\mathrm{b}}$, Ronald W. Raab ${ }^{\mathrm{b}}$, Alan C. Rapraeger ${ }^{\mathrm{c}}$, and Gordon W. \\ Laurie $^{\mathrm{a},{ }^{*}}$ \\ a Department of Cell Biology, University of Virginia, P.O. Box 800732 UVa Health System, \\ Charlottesville VA, 22908-0732 USA \\ b Department of Integrated Science and Technology, James Madison University, Harrisonburg \\ VA, 22807 USA \\ c Department of Pathology and Laboratory Science, University of Wisconsin-Madison, Madison \\ WI 53706 USA
}

\section{Keywords}

dry eye; cornea; SDC1; HPSE; LACRT

\section{Structure}

Syndecan-1 (NP_001006947.1) is a widely expressed cell surface heparan sulfate proteoglycan whose molecular cloning as 'syndecan' (Saunders et al, '89), later known as syndecan-1, was the springboard for the discovery of syndecans-2 through -4. Expression in the eye has been documented in corneal, limbal (GDS2433) and conjunctival epithelial cells, meibomian (GDS1361) and lacrimal (NEIBank) glands, and in retina and retinal pigmented epithelium/choroid (NEIBank). The syndecan-1 gene 'SDC1' consists of five exons extending over $24.6 \mathrm{~kb}$ in human chromosome 2. Ensembl release 57 lists thirty-four orthologues ranging from sea squirt to human. After cleavage of the signal peptide, syndecan-1 in humans is 288 amino acids long with a theoretical molecular weight and pI respectively of $30 \mathrm{kDa}$ and 4.5. Migration in SDS PAGE however appears as a high molecular weight smear because glycosylation is extensive with substitution by as many as three heparan sulfate and two chondroitin sulfate glycosaminoglycans (Fig. 1). Heparan sulfate polymerization is initiated on serines 37 ( $15^{*}$ [*after signal peptide cleavage]), 45 $\left(23^{*}\right)$ and $47\left(25^{*}\right)$ within the respective amino terminal core protein sequences DG $\underline{S} G D$ and NFSGGSA. Chondroitin sulfate can be substituted on serines 207 (185*; EG $\underline{S} G E)$ and 217 (195*; ETSGE) in proximity with the ectodomain cleavage site RKE and the adjacent transmembrane domain. One N-linked glycosylation site is predicted on asparagine 43 (22*; NFSG; NetNGlyc 1.0). NetOGlyc 3.1 reveals 33 potential sites of O-glycosylation - most between between amino acids $68\left(46^{*}\right)$ and $180\left(158^{*}\right)$ (Fig. 1). The human core protein sequence is subject to some variation with four known nonsynonymous single nucleotide polymorphisms: proline 19 to leucine within the signal peptide, threonine $75\left(53^{*}\right)$ to methionine, leucine $136(114 *)$ to glutamine, and glycine 259 (237*) to valine (Fig. 1). Variation is also introduced at the level of alternative splicing. Variant transcripts code for mature core proteins of 169, 215 and 288 amino acids, the latter translated as the wild type

*Corresponding author glaurie@ virginia.edu (G.W. Laurie).

Publisher's Disclaimer: This is a PDF file of an unedited manuscript that has been accepted for publication. As a service to our customers we are providing this early version of the manuscript. The manuscript will undergo copyediting, typesetting, and review of the resulting proof before it is published in its final citable form. Please note that during the production process errors may be discovered which could affect the content, and all legal disclaimers that apply to the journal pertain. 
form. The 169 amino acid variant is identical to the first 159 amino acids of wild type, and thus is a secreted heparan sulfate proteoglycan with a mature core protein of $15.3 \mathrm{kDa}(\mathrm{pI}$ 4.3). ESTs suggest that it is expressed in multiple tissues including the optic nerve and keratoconus cornea (Aceview). The 215 amino acid form is predicted to be cytoplasmic. Other transcript variants do not appear to code for proteins (Ensembl, Aceview).

\section{Function}

Syndecan-1 function is almost entirely dominated by its negatively charged heparan and chondroitin sulfate chains that bind and sequester cationic proteins and peptides. Heparan sulfate binds fibroblast (FGF), vascular endothelial and hepatocyte growth factors, glial cell derived neurotrophic factor, midkine, pleiotrophin, Wnts, noggin, fibronectin, laminins, collagens, thrombospondin and others. Chondroitin sulfate also binds midkine and pleiotrophin. Via glycosaminoglycan-growth factor interactions, syndecan-1 serves as an important co-receptor for receptor tyrosine kinases, and some G-protein coupled receptors. By ligating extracellular molecules, it serves as a receptor that can lead to signaling.

Extracellular enzymes, such as endo-6-O sulfatases 'SULF1' and '-2', play a critical role in modulating heparan sulfate binding specificity. The importance of sulfation first emerged from studies of FGF2-dependent growth and myoblast differentiation (Rapraeger et al '91). Other enzymes under inflammatory cytokine control attack the RKE cleavage site to promote ectodomain shedding. Shedding augments inflammation and diminishes wound healing, whereas intact syndecan-1 suppresses leukocyte binding of capillaries to minimize inflammation. Ectodomain shedding can also depress inflammation by minimizing the quantity of inflammatory cytokines sequestered by heparan sulfate. Another key modulating enzyme is heparanase, a heparin binding endo- $\beta$-D-glucuronidase that trims heparan sulfate. Trimming potentially exposes core protein sites. The ocular surface growth factor 'lacritin' in human tears is dependent on extracellular heparanase for activity (Ma et al, '06). A heparanase 'off/on' switch exposes or generates a lacritin binding site within the N-terminal 50 amino acids of syndecan-1. Bound lacritin, via a signaling receptor, then triggers mitogenesis of subconfluent corneal epithelial cells. A heparanase-independent $\alpha v \beta 3$ and $\alpha v \beta 5$ integrin targeting activity has been localized between amino acids $89(67 *)$ and 120 $\left(98^{*}\right)$ of syndecan-1. Full activity is encapsulated in the peptide mimic 'synstatin' with promise as an angiogenesis and tumorigenesis inhibitor (Beauvais et al, '09). Released heparan sulfate fragments may bind, or already be saturated with, growth factors. Released fragment/growth factor conjugates diffuse freely to target cellular receptors. Receptor binding affinity is often enhanced by heparan sulfate (Rapraeger et al, '91).

\section{Disease Involvement}

Syndecan-1 can bind and augment viral infectivity (ie. HIV). It also appears to play an important role in cancer progression, although expression may be increased or reduced, depending on specific cancer type or even within cancer types. Variables include tumor phenotype and syndecan function. Microarray analyses report numerous examples of twofold or greater SDC1 overexpression in bladder, brain, breast, lung, ovarian, pancreatic and testicular cancer. In melanoma, lymphoma, leukemia, and prostate cancer, the opposite is true (Oncomine 4.3). Although characterized as an epithelial syndecan, it also becomes highly expressed in the reactive stroma and lost in the epithelium of some breast tumors, a shift that may emphasize its role in regulating signaling between the stromal-epithelial compartments. Syndecan-1 is highly expressed and indicative of poor prognosis in multiple myeloma, especially when expressed in conjunction with heparanase, which upregulates syndecan-1 expression and induces ectodomain shedding that reaches high levels in patient 
sera. Decreased protein expression heparan sulfate biosynthetic enzymes in dry eye could contribute to ocular surface pathology.

\section{Future Studies}

Ligation of syndecan-1, $-2,-3$ and -4 core proteins and heparan sulfate chains, localize syndecans to sites of adhesion and growth factor signaling where they engage and stimulate signaling of other cell surface receptors. Syndecan gene family redundancy is largely unexplored. Combinatory contributions and redundancies could be addressed by combining syndecan- 1 to -4 gene knockouts in tissue specific systems. New studies should address the frequency and functional significance of syndecan-1 ectodomain shedding on the ocular surface, whether shed syndecan-1 plays a role in tear biophysics, the significance of epithelial-derived tear heparanase in 'off/on' switch regulation of lacritin function, and whether biosynthesis or modification of heparan sulfate is altered in dry eye.

\section{Acknowledgments}

Supported by NIH RO1 EY13143 and EY018222 to GWL, NIH R01 CA118839 and CA139872 to ACR and AHA 09GRNT2250572 to ACR.

\section{Abreviations
LACRT
lacritin
SDC1
syndecan-1

\section{References}

Beauvais DM, Ell BJ, McWhorter AR, Rapraeger AC. Syndecan-1 regulates alphavbeta3 and alphavbeta5 integrin activation during angiogenesis and is blocked by synstatin, a novel peptide inhibitor. J Exp Med. 2009; 206:691-705. [PubMed: 19255147]

Ma P, Beck SL, Raab RW, McKown RL, Coffman GL, Utani A, Chirico WJ, Rapraeger AC, Laurie GW. Heparanase deglycanation of syndecan-1 is required for binding of the epithelial-restricted prosecretory mitogen lacritin. J Cell Biol. 2006; 74:1097-1106. [PubMed: 16982797]

Rapraeger AC, Krufka A, Olwin BB. Requirement of heparan sulfate for bFGF- mediated fibroblast growth and myoblast differentiation. Science. 1991; 252:1705-1708. [PubMed: 1646484]

Saunders S, Jalkanen M, O'Farrell S, Bernfield M. Molecular cloning of syndecan, an integral membrane proteoglycan. J Cell Biol. 1989; 108:1547-1556. [PubMed: 2494194] 


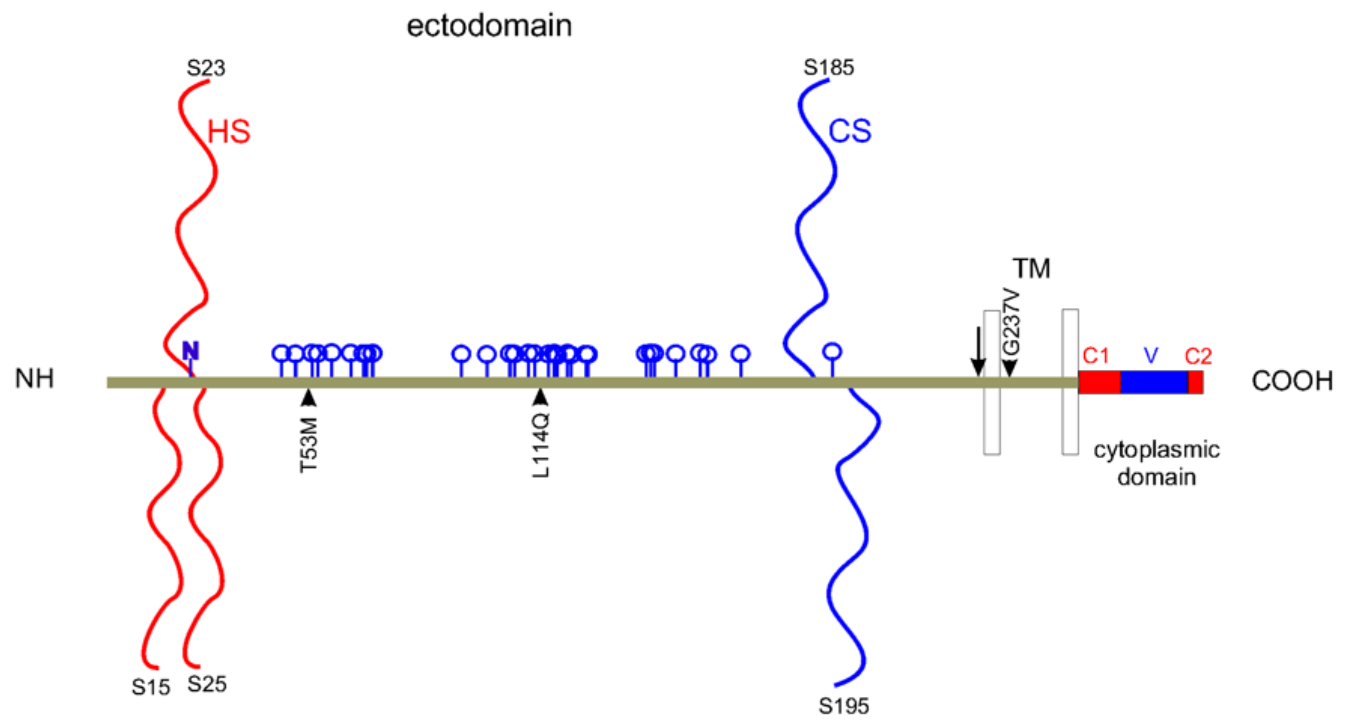

Figure 1.

Structural features of syndecan-1. Linear model of human syndecan-1, excluding the signal peptide, with heparan (HS) and chondroitin (CS) sulfate chains, predicted N- (N) and O(blue pegs) linked glycosylation, non-synonymous variation (arrowheads), ectodomain cleavage site (vertical arrow), transmembrane (TM) and cytoplasmic domains (with $\mathrm{C} 1, \mathrm{~V}$ and $\mathrm{C} 2$ regions). 